Obere Extremität 2021 · 16:286-288 https://doi.org/10.1007/s11678-021-00660-x Received: 26 April 2021 Accepted: 15 June 2021

Published online: 22 September 2021

(c) The Author(s) 2021

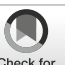

\section{"Parachute" technique for combined irreparable subscapularis and posterosuperior rotator cuff tears}

\author{
Bassem T. Elhassan · Khang H. Dang · Tiffany M. Huynh (D) \\ Harvard Shoulder Service, Massachusetts General Hospital, Boston, USA
}

\section{Background}

Irreparable massive rotator cuff tears in young and active patients remain challenging for shoulder surgeons. Rotator cuff tears are considered irreparable if there is retraction to the glenoid, a tendon length less than $1 \mathrm{~cm}$, fatty infiltration of Goutallier grade 2 or greater, and/or advanced muscle atrophy $[1,5]$. Repair of these tears commonly fails, with an incidence of rerupture of up to $94 \%$ [4].

Although reverse shoulder arthroplasty (RSA) may be an option, it is not a durable solution for young, active patients due to concerns about implant longevity and joint preservation. In this population, tendon transfers may be more appropriate as they may restore shoulder function and anatomic joint mechanics. This may prevent the development of osteoarthritis due to eccentric joint loading.

The "parachute" technique involves the transfer of two tendons, the lower trapezius (LT) and the latissimus dorsi (LD), to reconstruct the shoulder in the context of concurrent irreparable subscapularis and posterosuperior rotator cuff tears. The procedure may be performed either open or arthroscopically assisted; this article details the arthroscopic-assisted technique.

Indications for the parachute technique include (1) pain and limitation in shoulder function that interferes with daily activities; (2) magnetic resonance imaging evidence of a combined irreparable subscapularis and posterosuperior rotator cuff tear; (3) normal function of the LT and LD muscles; and (4) minimal or no arthritis. The technique may address functional deficits in multiple planes including external rotation, internal rotation, and pseudoparalysis.

\section{Operative technique}

The parachute technique is performed with the patient in the beach chair position and under general anesthesia. A dynamic arm holder is used, and the patient is draped such that the entire upper extremity and hemithorax are accessible. Tendon harvesting and Achilles tendon allograft preparation are performed first before the arthroscopic portion of the procedure to minimize swelling.

\section{Achilles tendon allograft preparation}

An Achilles tendon allograft is used to augment the LT tendon transfer. After sharply removing the calcaneal bone block, the medial and lateral edges of the tendon are prepared with no. 2 nonabsorbable sutures for approximately $5 \mathrm{~cm}$ in a Krakow fashion. One side of the allograft is colored with a skin marker to designate it as the dorsal side. Until the prepared allograft is used, it is soaked in normal saline with $1 \mathrm{~g}$ vancomycin to decrease the risk of infection. If an autograft tendon is preferred, an alternative to the Achilles tendon allograft may be a hamstring autograft, prepared in either a single- or double-stranded fashion. Reasons for using an autograft tendon include cost, lack of access to allograft material, and surgeon preference. 
Harvest of the latissimus dorsi tendon

The shoulder is positioned in abduction and internal rotation to expose the axilla. An inverted L-shaped incision is made over the posterior axillary line, where the underlying LD is palpable. At this level, the tendinous portion of the $L D$ is encountered superficially, and the muscular teres major (TM) lies deeper. Separate the LT tendon from the TM and sharply release it from its insertion on the humerus. Prepare the tendon end with two no. 2 nonabsorbable sutures in a Krakow fashion and color its dorsal aspect with a skin marker. Dissect the fascial adhesions off the LT tendon to the level of the scapula to maximize excursion.

\section{Harvest of the lower trapezius tendon}

The following landmarks on the scapula are marked: (1) the scapular spine, (2) medial border of the scapular body, (3) LT insertion on the scapular spine, and (4) acromion. A horizontal incision is made just inferior to the scapular spine, extending from $1 \mathrm{~cm}$ medial and approximately $4 \mathrm{~cm}$ lateral to the medial scapular border. After the skin flaps are raised, the fascia overlying the LT is encountered. Grasp the layer of fat overlying the LT tendon and excise it with electrocautery to expose the triangularshaped tendon.

Detach the LT tendon off the scapular spine and mobilize it from the underlying infraspinatus fascia. It is important to avoid the spinal accessory nerve, which runs deep to the LT tendon, slightly medial to the scapular body. Prepare the superior and inferior edges of the tendon with no. 2 nonabsorbable sutures in a Krakow fashion. Excise a portion of the infraspinatus fascia to allow for later passage of the Achilles tendon allograft.

\section{Arthroscopic latissimus dorsi tendon transfer}

Anterolateral, anterior, and distal anterior arthroscopic portals are used to transfer the LD tendon. The lesser tuberosity is debrided of remaining soft tissue to allow for tendon healing.
With the arthroscope in the anterolateral portal, visualize the triangular interval between the humerus laterally, the conjoint tendon medially, and the pectoralis major anteriorly. To confirm the correct interval, place a finger into the axillary wound and palpate the prior LD insertion. One can visualize the finger in the triangular interval arthroscopically. Place a long grasping instrument into the distal anterior portal and out of the axillary incision. Grasp the sutures of the prepared LD tendon to pass it to the anterior aspect of the shoulder. Then, attach the LD tendon to the lesser tuberosity using two suture anchors via the anterior portals.

\section{Scope-assisted lower trapezius transfer}

Standard anterior, lateral, posterolateral, and Neviaser arthroscopic portals are used to transfer the LT tendon. While visualizing from the lateral portal, debride the great tuberosity of remaining soft tissue. Create a Neviaser portal to place a suture anchor on the medial aspect of the greater tuberosity, just lateral to the articular cartilage. Clamp the sutures, which will later be used to compress the transferred tendon in a medial-to-lateral direction over the greater tuberosity.

Place a long grasper in the anterior portal and toward the posterior incision, where the lower trapezius tendon was harvested. The grasper should pass between the infraspinatus and deltoid interval and should be visualized exiting the previously excised infraspinatus fascia. Use the grasper to pass the sutures of the previously prepared Achilles tendon allograft into the shoulder joint.

Using the anterior portal, attach the sutures of the Achilles tendon allograft onto the anterolateral and anteromedial aspects of the greater tuberosity with two suture anchors. Then, visualize from a posterolateral portal to retrieve sutures from the Neviaser portal and out the lateral portal. Secure these sutures to the lateral aspect of the greater tuberosity with another suture anchor, such that the sutures form a compressive bridge over the Achilles tendon allograft. This will compress the allograft to the humeral head and prevent bowstringing.
Attention is then brought to the posterior scapular incision to secure the Achilles allograft onto the LT tendon. Position the shoulder in $90^{\circ}$ of abduction and $90^{\circ}$ of external rotation. Longitudinally split the Achilles tendon allograft and excise the superior half, since it is not needed. Pass the Achilles tendon allograft through the LT tendon in a Pulvertaft fashion. While an assistant pulls with tension on the ends of the LT tendon and Achilles tendon allograft, suture the tendons together with no. 2 nonabsorbable sutures. Take care not to suture the deltoid or underlying infraspinatus to the construct as this may limit excursion. Redundant Achilles tendon allograft is resected, and wounds are closed.

\section{Postoperative rehabilitation protocol}

Place the shoulder in an immobilizer in approximately $45-60^{\circ}$ of abduction and neutral rotation for 8 weeks. After 8 weeks, the immobilizer is removed, and the patient may return to most activities of daily living. Additionally, the patient may start physical therapy with active assisted range of motion exercises and aquatherapy. At 16 weeks after surgery, the patient may start progressive stretching and strengthening exercises in all planes. The patient may participate in full activities without limitations 6 months after surgery.

\section{Discussion}

The management of irreparable massive rotator cuff tears is challenging, especially in young active patients. In this population, tendon transfers offer a reliable alternative to less durable options such as RSA, arthroscopic debridement, partial rotator cuff repair, and interpositional graft constructs.

The parachute technique involves the transfer of the LT and LD tendons for irreparable combined subscapularis and posterosuperior rotator cuff tears. Lower trapezius tendon transfers have been described by Elhassan et al. to reconstruct irreparable posterosuperior rotator cuff tears. It is the ideal donor tendon as it possesses a similar line of pull, excursion, and tension as the infraspinatus [1]. Elhassan 
et al. have described LD tendon transfers to reconstruct irreparable anterosuperior tears, since the tendon has a similar line of pull as the subscapularis [2]. Studies on the outcomes of LT or LD tendon transfers demonstrate that these procedures restore shoulder function and improve pain levels [1-3]. Most importantly, they may restore anatomic joint mechanics and preserve the patient's native glenohumeral joint.

Prior to the development of the parachute technique, a tendon transfer for external rotation was only recommended if the patient had functional internal rotation and vice versa. This was because the patient would continue to experience a functional deficit postoperatively since single tendon transfers only address either external or internal rotation. With the parachute technique, two tendon transfers are utilized to address multiple planes of motion simultaneously. Consequently, functional deficits in both external and internal rotations would be an indication for the procedure, rather than a contraindication.

Potential complications include seroma formation, infection, impingement of the allograft in the subacromial space, and rupture of the tendon transfers. A forthcoming publication, by the authors of this technical note, details the outcome of the parachute technique. Patients are expected to have significant improvement in their patientreported outcome measures and range of motion, particularly with external rotation. Patients with pseudoparalysis experienced resolution, except those with fixed anterosuperior escape preoperatively; this patient population only experienced improved rotation and may benefit from reverse shoulder arthroplasty.

\section{Practical conclusion}

- The "Parachute" technique is a combination of two tendon transfers, the lower trapezius and latissimus dorsi, which alleviates pain and restores shoulder function.

- It is a reliable joint-preserving option in young, active patients with irreparable subscapularis and posterosuperior rotator cuff tears.

\section{Corresponding address}

\section{Bassem T. Elhassan, MD}

Harvard Shoulder Service, Massachusetts

General Hospital

55 Fruit Street, 02114 Boston, MA, USA

belhassan@partners.org

\section{Declarations}

Conflict of interest. B.T. Elhassan, K.H. Dang, and T.M. Huynh declare that they have no competing interests.

For this article no studies with human participants or animals were performed by any of the authors. All studies performed were in accordance with the ethical standards indicated in each case.

Open Access. This article is licensed under a Creative Commons Attribution 4.0 International License, which permits use, sharing, adaptation, distribution and reproduction in any medium or format, as long as you give appropriate credit to the original author(s) and the source, provide a link to the Creative Commons licence, and indicate if changes were made. The images or other third party material in this article are included in the article's Creative Commons licence, unless indicated otherwise in a credit line to the material. If material is not included in the article's Creative Commons licence and your intended use is not permitted by statutory regulation or exceeds the permitted use, you will need to obtain permission directly from the copyright holder. To view a copy of this licence, visit http://creativecommons.org/licenses/by/4.0/.

\section{References}

1. Elhassan BT, Sanchez-Sotelo J, Wagner ER (2020) Outcome of arthroscopically assisted lower trapezius transfer to reconstruct massive irreparable posterior-superior rotator cuff tears. J Shoulder Elbow Surg 29:2135-2142. https://doi.org/10. 1016/j.jse.2020.02.018

2. Elhassan BT, Wagner ER, Kany J (2020) Latissimus dorsi transfer for irreparable subscapularis tear. J Shoulder Elbow Surg 29:2128-2134. https://doi. org/10.1016/j.jse.2020.02.019

3. Elhassan BT, Wagner ER, Werthel J-D (2016) Outcome of lower trapezius transfer to reconstruct massive irreparable posterior-superior rotator cuff tear. JShoulder Elbow Surg 25:1346-1353. https:// doi.org/10.1016/j.jse.2015.12.006

4. Galatz LM, Ball CM, Teefey SA et al (2004) The outcome and repair integrity of completely arthroscopically repaired large and massive rotator cuff tears. J Bone Joint Surg Am 86:219-224. https://doi.org/10.2106/00004623-20040200000002

5. Goutallier D, Postel JM, Bernageau J et al (1994) Fatty muscle degeneration in cuff ruptures. Preand postoperative evaluation by CT scan. Clin Orthop Relat Res 304:78-83 